\title{
A Splitting Algorithm for Medical Image Denoising
}

\author{
Adérito Araújo \\ CMUC, Department of Mathematics, University of Coimbra, 3000 Coimbra, Portugal \\ alma@mat.uc.pt
}

Keywords: $\quad$ Finite Differences, Optical Coherence Tomography, Image Denoising.

\begin{abstract}
In this work we consider a stable algorithm for integrating a mathematical model based on mean curvature motion equation proposed in (Alvarez, Lions, Morel 1992) for image denoising. The scheme is constructed using a finite difference space discretisation and semi-implicit time discretisation and is considered with a splitting algorithm that can be implemented in parallel. We apply this algorithm to the problem of denoising optical coherence tomograms from the human retina while preserving image features.
\end{abstract}

\section{INTRODUCTION}

Optical coherence tomography (OCT) is a noninvasive imaging modality with an increasing number of applications and it is becoming an essential tool in ophthalmology allowing in vivo high-resolution cross-sectional imaging of the retinal tissue. It relies in certain optical characteristics of light to provide information of the eye fundus, facilitating the diagnosis of several eye pathologies such as macular degeneration, cone-rod dystrophy, retinopathy and glaucoma (Junqueira, Carneiro 2005). All these pathologies can be diagnosed more conclusively with the help OCT(Serranho, Morgado, Bernardes 2012), (Bouma, Tearney 2002). In fact, previous studies have established a link between changes in the blood-retina barrier and in optical properties of the retina (Bernardes, Santos, Serranho, Lobo, Cunha-Vaz 2011) which can be identified by this exam.

As any imaging technique that bases its image formation on coherent waves, OCT images suffer from speckle noise, which reduces its quality. Despeckling optical coherence tomograms from the human retina is a fundamental step to a better diagnosis or as a preprocessing stage for retinal layer segmentation (Bernardes, Maduro, Serranho, Araújo, Barbeiro Cunha-Vaz 2010). Both of these applications are particularly important in monitoring the progression of retinal disorders.

Physically, OCT is based in low coherence interferometry. This technique uses an electromagnetic wave with a low coherence length (meaning the wave is coherent, i.e., highly self-correlated, in a small space interval). The light beam emitted from the source is split into two identical beams with a beam splitter (see Figure 1). Then, while one of the resulting waves (i.e. light beam) travels to a reference mirror and back, the other goes to a sample and is reflected by structures there present. These reflected waves recombine at the splitter. The portions of the waves that are coherent interfere with each other, resulting in an interference pattern which yields information about the sample at a given depth (Bernardes, Cunha-Vaz, Serranho 2012), (Bouma, Tearney 2002).

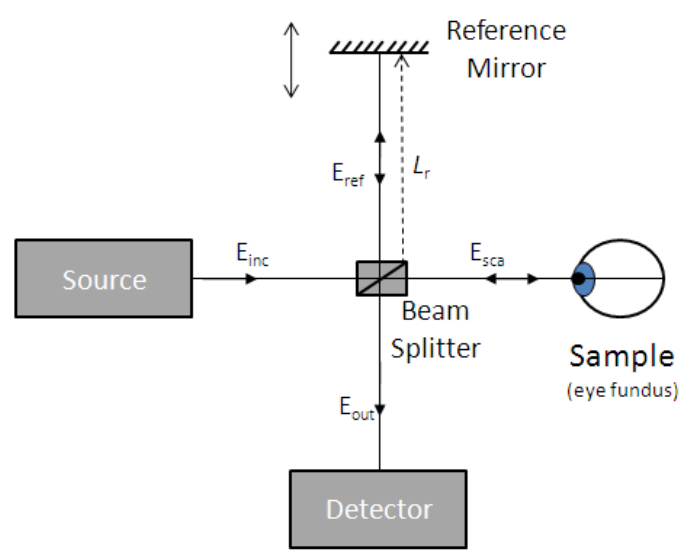

Figure 1: Schematic of the optical coherence tomography apparatus.

The main purpose of this work is to consider an algorithm to reduce the speckle noise for both the visual assessment and the improved structure segmentation on high- definition spectral domain Cirrus OCT (Carl Zeiss Meditec, Dublin, CA, USA). This reti- 
nal imaging system allows the acquisition of volumes of $200 \times 1024$ or $512 \times 128 \times 1024$ voxels, respectively, for the lateral, azimuthal and axial directions (Figure 2). These volumetric data are obtained from a $6000 \times 6000 \times 2000 \mu \mathrm{m}^{3}$ volume of the human macula. Additionally, high- resolution B-scan images of $1024 \times 1024$ pixels can be obtained.
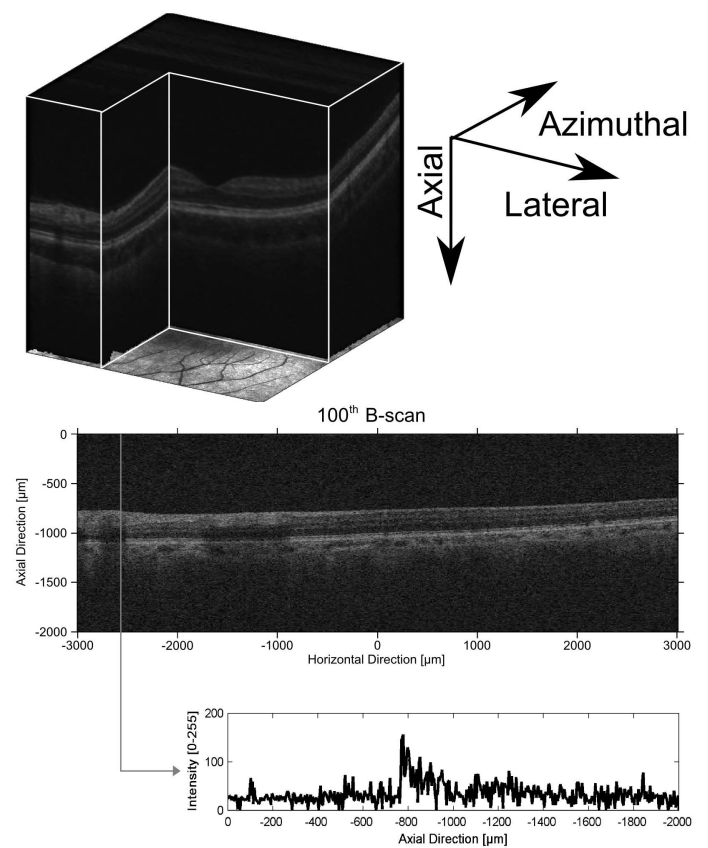

Figure 2: Optical coherence tomography (OCT). Top: volumetric OCT data shown over an eye fundus reference. Bottom example of a B-scan (top) and an A-scan profile (bottom).

The paper is organised as follows. In Section 2 we present the mathematical model for image denoising. In Section 3 we define the finite difference implicitexplicit scheme that can be implemented in parallel and prove that the algorithm is stable with respect to the infinity norm. In Section 3 we consider the application of the proposed filter to an example with synthetic as well as to an OCT high-resolution B-scan from the human eye fundus. We finish with some conclusions.

\section{MATHEMATICAL MODEL}

Let $\phi \in C^{2}(\Omega \times[0, T])$ with $\Omega \subset \mathbb{R}^{2}$ a compact set and $\phi_{0} \in C(\Omega)$. We consider the problem

$$
\left\{\begin{array}{r}
\phi_{t}=g\left(\left|\nabla G_{\sigma} * \phi\right|\right)|\nabla \phi| \operatorname{div}\left(\frac{\nabla \phi}{|\nabla \phi|}\right), \\
(x, y) \in \Omega, t \in] 0, T] \\
\phi(x, y, 0)=\phi_{0}(x),(x, y) \in \Omega \\
\phi(x, y, t)=0,(x, y) \in \partial \Omega, t \in[0, T]
\end{array}\right.
$$

where $\frac{\partial \phi}{\partial v}$ denotes the derivative in the direction of the exterior normal to $\partial \Omega, G_{\sigma}$ a smoothing kernel that depends on a parameter $\sigma$ (e.g. a Gaussian) and $g(s)$ is a nondecreasing real function which tends to zero as $s \rightarrow \infty$. This problem was proposed in (Alvarez, Lions, Morel 1992) for image smooting and edge detection where $\phi_{0}(x, y)$ represents the try level of the original noisy image, $\phi(x, y, t)$ is its smoothed version depending on the scale parameter $t$. The term $|\nabla \phi| \operatorname{div}\left(\frac{\nabla \phi}{|\nabla \phi|}\right)$ represents a degenerate diffusion term, which diffuses $\phi$ in the direction to its gradient. The term $g\left(\left|\nabla G_{\sigma} * \phi\right|\right)$ is used for the enhancement to the edges, since it controls the speed of the diffusion: if the gradient of $\phi$ has a small mean in a neighbourhood of a point, this point is considered the interior point of a smooth region of the image and the diffusion is strong; if the gradient has a large mean value on the neighbourhood of a point, this point is considered an edge point and the diffusion spread is lowered since $g(s)$ is small for large $s$.

The equation (1) is difficult to study since, besides its non-linearity, is not defined in the points where $|\nabla \phi|=0$. In order to prevent the situation of possible zero gradients, we will consider the Evans-Spruck type regularization (Evans, J. Spruck 1991) and consider $\left|\nabla_{\varepsilon} \phi\right|$ instead of $|\nabla \phi|$, where

$$
\nabla_{\varepsilon} \phi=\left(\nabla^{T} \phi, \varepsilon\right)^{T}
$$

with $0 \leq \varepsilon \ll 1$. In other words, we replace $|\nabla \phi|$ by

$$
\left|\nabla_{\varepsilon} \phi\right|=\sqrt{|\nabla \phi|+\varepsilon^{2}}
$$

in (1) obtaining the modified problem that we will write in the form

$$
\left\{\begin{array}{r}
\frac{\phi_{t}}{g\left(\left|\nabla G_{\sigma} * \phi\right|\right)\left|\nabla_{\varepsilon} \phi\right|}=\operatorname{div}\left(\frac{\nabla \phi}{\left|\nabla_{\varepsilon} \phi\right|}\right), \\
(x, y) \in \Omega, t \in] 0, T] \\
\phi(x, y, 0)=\phi_{0}(x),(x, y) \in \Omega \\
\phi(x, y, t)=0,(x, y) \in \partial \Omega, t \in[0, T]
\end{array}\right.
$$


Note that, for zero gradients, this problem reduces to the heat equation, which is suitable for smoothing purposes. On the other hand, for large values of the gradient, the influence of $\varepsilon$ can be neglected.

\section{MUMERICAL METHOD}

\subsection{A Linearly Implicit Finite Difference Scheme}

Let $\Delta t>0$ and $t^{n}=n \Delta t$, with $n=0, \ldots, N$ such that $t_{0}=0$ and $t_{N}=T$ and $\left.\Omega=\right] 0, X[\times] 0, Y\left[, N_{x}, N_{y} \in \mathbb{N}\right.$ and $h>0$ such that

$$
h=\frac{X}{N_{x}}=\frac{Y}{N_{y}} .
$$

Let us also consider $x_{i}=i h$ and $y_{j}=j h$, for $i=$ $0,1, \ldots, N_{x}$ and $j=0,1, \ldots, N_{y}$. These points define a rectangular grid that we denote by

$$
\bar{\Omega}_{h}=\left\{\left(x_{i}, y_{j}\right): i=0,1, \ldots, N_{x}, j=0,1, \ldots, N_{y}\right\} .
$$

Let $\phi_{i j}^{n} \approx \phi\left(x_{i}, y_{j}, n \Delta t\right)$ denote the solution of the finite differences problem

$$
\begin{aligned}
\frac{1}{g_{i j}^{n}\left|\nabla_{\varepsilon, h} \phi_{i j}^{n}\right|} \frac{\phi_{i j}^{n+1}-\phi_{i j}^{n}}{\Delta t}= & D_{x}^{+}\left(\frac{D_{x}^{-} \phi_{i j}^{n+1}}{\left|\nabla_{\varepsilon, h} \phi_{i j}^{n}\right|}\right) \\
& +D_{y}^{+}\left(\frac{D_{y}^{-} \phi_{i j}^{n+1}}{\left|\nabla_{\varepsilon, h} \phi_{i j}^{n}\right|}\right)
\end{aligned}
$$

in which

$$
\begin{gathered}
g_{i j}^{n}=g\left(\left|\nabla G_{\sigma} * \phi_{i j}^{n}\right|\right), \\
\nabla_{\varepsilon} \phi=\left(D_{x}^{-} \phi_{i j}^{n}, D_{y}^{-} \phi_{i j}^{n}, \varepsilon\right)^{T},
\end{gathered}
$$

and

$$
\left|\nabla_{\varepsilon, h} \phi_{i j}^{n}\right|=\sqrt{\left(D_{x}^{-} \phi_{i j}^{n}\right)^{2}+\left(D_{y}^{-} \phi_{i j}^{n}\right)^{2}+\varepsilon^{2}}
$$

with the first order finite differences operators defined by

$$
\begin{aligned}
& D_{x}^{-} U_{i j}=\frac{U_{i j}-U_{i-1, j}}{h}, i=1, \ldots, N_{x}, j=1, \ldots, N_{y}-1, \\
& D_{x}^{+} U_{i j}=\frac{U_{i+1, j}-U_{i j}}{h}, i=0, \ldots, N_{x}-1, j=1, \ldots, N_{y}-1, \\
& D_{y}^{-} U_{i j}=\frac{U_{i j}-U_{i, j-1}}{h}, i=1, \ldots, N_{x}-1, j=1, \ldots, N_{y}, \\
& D_{y}^{+} U_{i j}=\frac{U_{i, j+1}-U_{i j}}{h}, i=1, \ldots, N_{x}-1, j=0, \ldots, N_{y}-1 .
\end{aligned}
$$

\subsection{A splitting algorithm}

The main idea behind splitting algorithms is to split the problem we want to solve in several simpler subproblems, independent or not, conveniently chosen.

Let $S$ be a space of functions and $\mathcal{A}$ an operator defined on $S$. Let us consider the equation

$$
\left.\left.\frac{\partial \phi}{\partial t}=\mathcal{A}(t, \phi)+f(t) \text { in } \Omega \times\right] 0, T\right], \phi(0)=\phi_{0} \in S .
$$

Let us now suppose that $\mathcal{A}$ and $f$ can be decomposed in the following way

$$
\mathcal{A}=\mathcal{A}_{1}+\cdots+\mathcal{A}_{m} \quad \text { and } \quad f=f_{1}+\cdots+f_{m} .
$$

Splitting algorithms take advantage of this decomposition, considering the $m$ subproblems

$$
\left.\left.\frac{\partial \phi}{\partial t}=\mathcal{A}_{k}(t, \phi)+f_{k}(t) \text { in } \Omega \times\right] 0, T\right], k=1, \ldots, m .
$$

Considering the time step $\Delta t, t_{n}=n \Delta t$, for $n=$ $1, \ldots, N$, and $\phi^{n}=\phi\left(x, t_{n}\right)$, with $\phi^{0}=\phi_{0}$, in (Lu, Neittaanmaki, Tai 1992) the authors proposed the following parallel algorithm:

At each level time $n=0, \ldots, N-1$ compute:

1. $\frac{\phi^{n+\frac{k}{2 m}}-\phi^{n}}{m \Delta t}=A_{k} \phi^{n+\frac{k}{2 m}}+f_{k}\left(\left(n+\frac{1}{2}\right) \Delta t\right)$,

2. $\phi^{n+1}=\frac{1}{m} \sum_{k=1}^{m} \phi^{n+\frac{k}{2 m}}$.

We pretend to consider the same approach to define a splitting algorithm for the discretized equation (3). Let us consider $\mathcal{A}=\mathcal{A}_{1}+\mathcal{A}_{2}$ with:

$$
\mathcal{A}_{1}\left(\phi^{n+1}\right)=D_{x}^{+}\left(\frac{D_{x}^{-} \phi_{i j}^{n+1}}{\left|\nabla_{\varepsilon, h} \phi_{i j}^{n}\right|}\right)
$$

and

$$
\mathcal{A}_{2}\left(\phi^{n+1}\right)=D_{y}^{+}\left(\frac{D_{y}^{-} \phi_{i j}^{n+1}}{\left|\nabla_{\varepsilon, h} \phi_{i j}^{n}\right|}\right) .
$$

We may then define the following sub-equations

$$
\frac{1}{g_{i j}^{n}\left|\nabla_{\varepsilon, h} \phi_{i j}^{n}\right|} \frac{\phi_{i j}^{n+\frac{1}{4}}-\phi_{i j}^{n}}{2 \Delta t}=\mathcal{A}_{1}\left(\phi^{n+1}\right)
$$

and

$$
\frac{1}{g_{i j}^{n}\left|\nabla_{\varepsilon, h} \phi_{i j}^{n}\right|} \frac{\phi_{i j}^{n+\frac{1}{4}}-\phi_{i j}^{n}}{2 \Delta t}=\mathcal{A}_{2}\left(\phi^{n+1}\right) .
$$


Let us consider the first equation written in the form

$$
\begin{gathered}
\frac{1}{g_{i j}^{n}\left|\nabla_{\varepsilon, h} \phi_{i j}^{n}\right|} \frac{\phi_{i j}^{n+\frac{1}{4}}-\phi_{i j}^{n}}{2 \Delta t}=\frac{\phi_{i-1, j}^{n+\frac{1}{4}}}{h^{2}\left|\nabla_{\varepsilon, h} \phi_{i, j}^{n}\right|} \\
-\frac{2}{h^{2}} \phi_{i j}^{n+\frac{1}{4}}\left(\frac{1}{\left|\nabla_{\varepsilon, h} \phi_{i+1, j}^{n}\right|}+\frac{1}{\left|\nabla_{\varepsilon, h} \phi_{i, j}^{n}\right|}\right) \\
+\frac{\phi_{i+1, j}^{n+\frac{1}{4}}}{h^{2}\left|\nabla_{\varepsilon, h} \phi_{i+1, j}^{n}\right|} .
\end{gathered}
$$

Let $\Phi^{n}$ the $\left(N_{y}-1\right)\left(N_{x}-1\right)$-dimensional vector

$$
\begin{aligned}
\Phi^{n}= & {\left[\phi_{1,1}^{n}, \ldots, \phi_{N_{x}-1,1}^{n}, \ldots, \phi_{1, j}^{n}, \ldots, \phi_{N_{x}-1, j}^{n}\right.} \\
& \left.\ldots, \phi_{1, N_{y}-1}^{n}, \ldots, \phi_{N_{x}-1, N_{y}-1}^{n}\right]^{T}
\end{aligned}
$$

and $A_{1}$ the $\left(N_{y}-1\right)\left(N_{x}-1\right) \times\left(N_{y}-1\right)\left(N_{x}-1\right)$ matrix with $\left(N_{y}-1\right)$ blocks of $\left(N_{x}-1\right) \times\left(N_{x}-1\right)$ tridiagonal matrices. The $j$-th block of $A_{1}, j=1, \ldots, N_{y}-1$, has entries

$$
\begin{gathered}
a_{i, i-1}=\frac{g_{i j}^{n}}{h^{2}} \\
a_{i, i}=-\frac{2 g_{i j}^{n}}{h^{2}}\left(\frac{\left|\nabla_{\varepsilon, h} \phi_{i, j}^{n}\right|}{\left|\nabla_{\varepsilon, h} \phi_{i+1, j}^{n}\right|}+1\right) \\
a_{i, i+1}=\frac{g_{i j}^{n}\left|\nabla_{\varepsilon, h} \phi_{i, j}^{n}\right|}{h^{2}\left|\nabla_{\varepsilon, h} \phi_{i+1, j}^{n}\right|}
\end{gathered}
$$

for $i=1, \ldots, N_{x}-1$. form

Then, the first sub-equation may be written in the

$$
\frac{\Phi^{n+\frac{1}{4}}-\Phi^{n}}{2 \Delta t}=A_{1} \Phi^{n+\frac{1}{4}} .
$$

Following the same approach, we conclude that the second sub-equation is equivalent to

$$
\frac{\Phi^{n+\frac{1}{2}}-\Phi^{n}}{2 \Delta t}=A_{2} \Phi^{n+\frac{1}{2}},
$$

where $A_{2}$ is a matrix of the same type as $A_{1}$ with entries

$$
\begin{gathered}
a_{k, k-\left(N_{y}-1\right)}=\frac{g_{i j}^{n}}{h^{2}}, \\
a_{k, k}=-\frac{2 g_{i j}^{n}}{h^{2}}\left(\frac{\left|\nabla_{\varepsilon, h} \phi_{i, j}^{n}\right|}{\left|\nabla_{\varepsilon, h} \phi_{i, j+1}^{n}\right|}+1\right), \\
a_{k, k+\left(N_{y}-1\right)}=\frac{g_{i j}^{n}\left|\nabla_{\varepsilon, h} \phi_{i, j}^{n}\right|}{h^{2}\left|\nabla_{\varepsilon, h} \phi_{i, j+1}^{n}\right|},
\end{gathered}
$$

where $k$ is related with $i, j$ by $k=(j-1)\left(N_{x}-1\right)+i$.

Since both $A_{1}$ and $A_{2}$ strictly diagonal dominant matrices, they are invertible and then we may define the following algorithm to solve (2) numerically:

At each level time $n=0, \ldots, N-1$ compute:
1. Compute, for $i=1, \ldots, N_{x}-1$ and $j=1, \ldots, N_{y}-1$,

$$
\left|\nabla_{\varepsilon, h} \phi_{i j}^{n}\right|=\sqrt{\left(D_{x}^{-} \phi_{i j}^{n}\right)^{2}+\left(D_{y}^{-} \phi_{i j}^{n}\right)^{2}+\varepsilon^{2}} ;
$$

2. Construct $A_{1}$ and $A_{2}$;

3. Solve

$$
\left(I-2 \Delta t A_{1}\right) \phi^{n+\frac{1}{4}}=\phi^{n}
$$

and

$$
\left(I-2 \Delta t A_{2}\right) \phi^{n+\frac{1}{2}}=\phi^{n}
$$

4. $\phi^{n+1}=\frac{\phi^{n+\frac{1}{4}}+\phi^{n+\frac{1}{2}}}{2}$.

With the properties of the matrices $A_{1}$ and $A_{2}$ we may prove that the algorithm is stable for the $\|\cdot\|_{\infty}$ norm. In fact, according to

$$
\begin{aligned}
\left|1+\frac{4 \Delta t g_{i j}^{n}}{h^{2}}\left(\frac{\left|\nabla_{\varepsilon, h} \phi_{i, j}^{n}\right|}{\left|\nabla_{\varepsilon, h} \phi_{i+1, j}^{n}\right|}+1\right)\right|< \\
\left|-\frac{2 \Delta t g_{i j}^{n}}{h^{2}}\right|+\left|-\frac{2 \Delta t g_{i j}^{n}\left|\nabla_{\varepsilon, h} \phi_{i, j}^{n}\right|}{h^{2}\left|\nabla_{\varepsilon, h} \phi_{i+1, j}^{n}\right|}\right|,
\end{aligned}
$$

the matrix $I-2 \Delta t A_{1}$ is strictly diagonal dominant. Since the diagonal entries of $I-2 \Delta t A_{1}$ are positive and the other entries are non-positive, we conclude that $I-2 \Delta t A_{1}$ is an M-matrix. In the same way, we conclude that $I-2 \Delta t A_{2}$ is also an M-matrix. Then there exists two positive constants $C_{1}$ and $C_{2}$ such that

$$
\left\|\left(I-2 \Delta t A_{i}\right)^{-1}\right\|_{\infty} \leq C_{i}, \quad i=1,2 .
$$

Then $\left\|\Phi^{n+\frac{1}{4}}\right\|_{\infty}+\left\|\Phi^{n+\frac{1}{2}}\right\|_{\infty} \leq\left(C_{1}+C_{2}\right)\left\|\phi^{n}\right\|_{\infty}$ and therefore

$$
\begin{aligned}
\left\|\Phi^{n+1}\right\|_{\infty} & \leq 2\left(\left\|\left(\Phi^{n+\frac{1}{4}}\left\|_{\infty}+\right\| \Phi^{n+\frac{1}{2}}\right)\right\|_{\infty}\right) \\
& \leq 2\left(c_{1}+c_{2}\right)\left\|\phi^{n}\right\|_{\infty}
\end{aligned}
$$

which proves the stability.

\section{NUMERICAL RESULTS}

In this section, we report our numerical testing for the proposed algorithm. In the Example 1, the basic features of the algorithm are explained. The second example is a two dimensional OCT image. In both examples we consider

$$
g(s)=\frac{1}{1+s^{2}}
$$

and

$$
G_{\sigma}(x, y)=\sigma^{-1 / 2} \exp \left(-\left|x^{2}+y^{2}\right| /(4 \sigma)\right) .
$$


Other choices for the function $g$ may be found in literature but this is the most commonly used in practical applications (Didas, Weickert 2007), (Plonka, Ma 2008).

For the proposed filter we consider a diffusion time of $0.3 \mathrm{~s}$. The filter was applied using 10 iterations with a time step $\Delta t=0.03 \mathrm{~s}$. The parameters for the gaussian kernel was $\sigma=1.5$. We also consider the spatial step size $h=1$ and the relaxation parameter $\varepsilon=10^{-5}$. The parameter $\varepsilon$ can be tuned in each application but it has no influence in the overall behavior of the algorithm.

Example 1 For our first test, we use the image shown in Figure 3. This image was obtained from a binary image by introducing $40 \%$ of salt and pepper noise. Salt and pepper noise presents a serious problem for segmentation algorithms that use image gradient information. This example, and others made with synthetic data, show that the algorithm preserves contrasts in the original image.
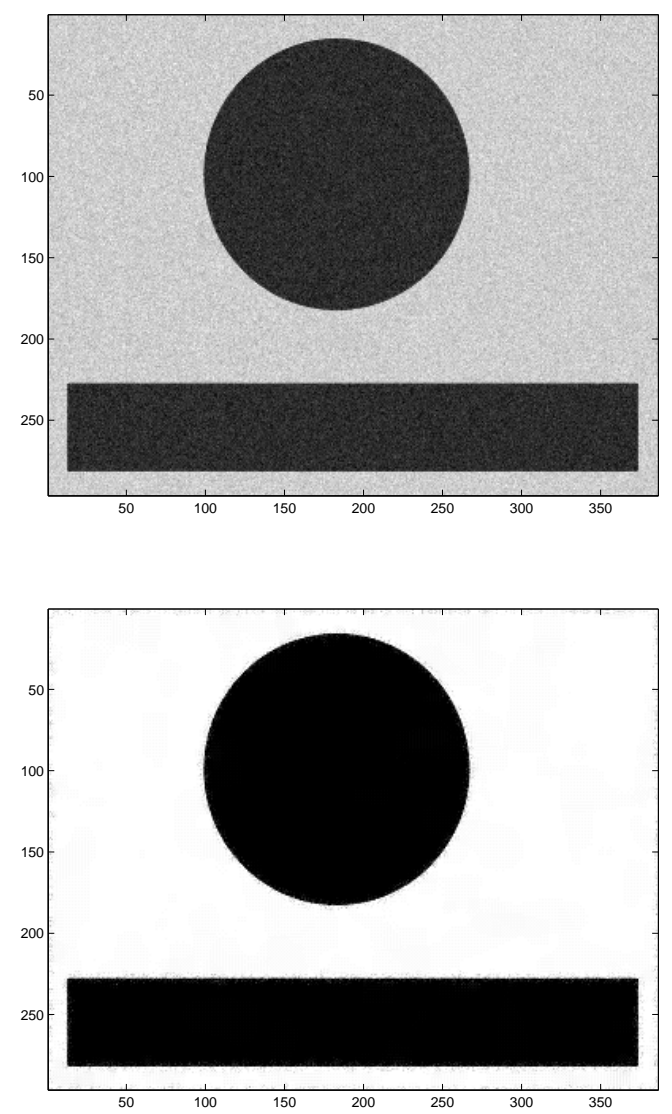

Figure 3: Top: Original test noisy image; bottom: denoised image.
Example 2 We consider a data set of images given by the Institute of Biomedical Research in Light and Image (IBILI), a research department of the Faculty of Medicine of the University of Coimbra, that correspond to real human eye fundus OCT data using the high-definition spectral domain Cirrus OCT (Carl Zeiss Meditec, Dublin, CA, USA). In our numerical tests, we considered $32 \mathrm{~B}$-scans using the macular cube protocol from 32 eye fundus scans from 13 healthy volunteers, 3 eyes with choroidal neovascularization, 2 with cystoid macular edema, 9 with diabetic retinopathy and 5 with age-related macular degeneration. In Figure 4 we just present one of these tests for a randomly chosen image.
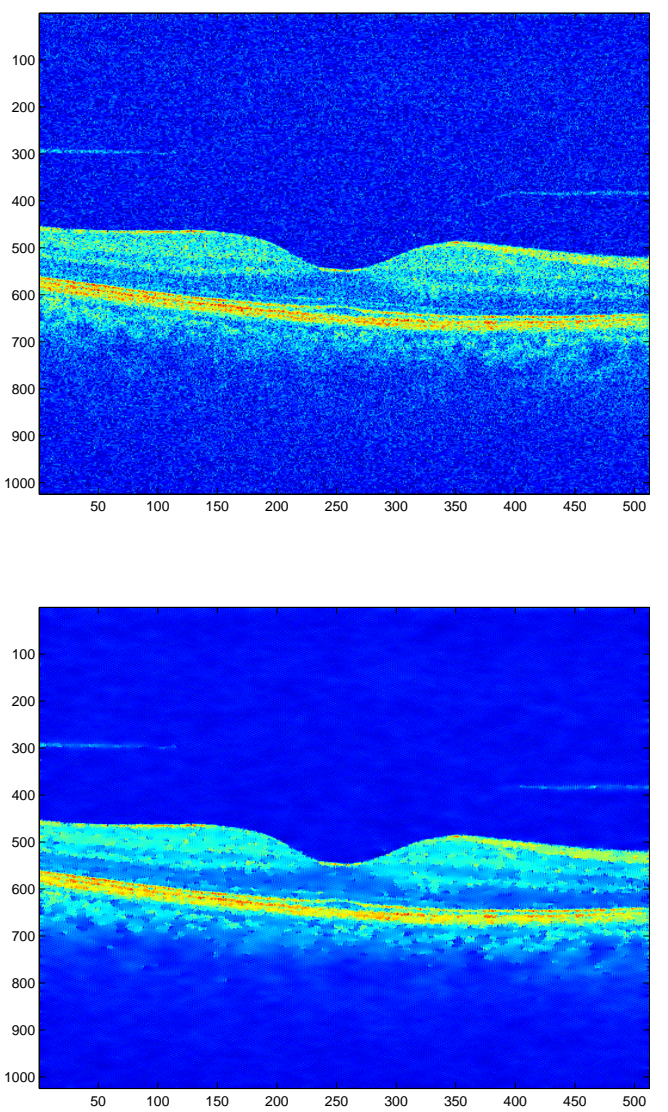

Figure 4: Top: Original OCT noisy image; bottom: denoised image.

Note the well-defined interface between the tissue and the vitreous regions. This allows us to conclude that the algorithm can be used not only to improve visual assessments of medical images but also as a preprocessor for image segmentation. 


\section{CONCLUSION AND FUTURE WORK}

In this work we consider an algorithm for integrating a mathematical model based on mean curvature motion equation proposed in (Alvarez, Lions, Morel 1992) for image denoising. The scheme has good stability properties and can be implemented in parallel. The application to despeckling optical coherence tomograms from the human retina show that the algorithm can be used as a preprocessing stage for OCT retina layer segmentation.

In the near future work we want to use wellknown speckle-reduction performance metrics (Salinas, Fernández 2007) to compare this algoritm with other filters, in particular with the nonlinear complex diffusion filter considered in (Bernardes, Maduro, Serranho, Araújo, Barbeiro Cunha-Vaz 2010). In addition to this particular area of application in the fundus of the human eye, this filter may be applied as well to different data sources corrupted with speckle noise, such as medical ultrasound.

\section{ACKNOWLEDGEMENTS}

This work has been partially supported by Centro de Matemática da Universidade de Coimbra (CMUC), funded by the European Regional Development Fund through the program COMPETE and by the Portuguese Government through the FCT - Fundação para a Ciência e a Tecnologia under the project PEst-C/MAT/UI0324/2011, and by FCT under the project PTDC/SAU-ENB/119132/2010) and COMPETE (QREN-FCOMP-01-0124-FEDER-021014) program. The author was also partially supported by program the project UTAustin/MAT/0066/2008.

\section{REFERENCES}

L. Alvarez, P-L Lions and J-M Morel (1992). Image Selective Smoothing and Edge Detection by Nonlinear Diffusion II. SIAM Journal on Numerical Analysis, 29(3), 845-866.

R. Bernardes, C. Maduro, P. Serranho, A. Araújo, S. Barbeiro, and J. Cunha-Vaz (2010). Improved adaptive complex diffusion despeckling filter. Optics Express, 18(23), 24048-24059.

R. Bernardes, T. Santos, P. Serranho, C. Lobo, and J. Cunha-Vaz (2011), Noninvasive evaluation of retinal leakage using OCT, Ophtalmologica, 226(2), 29-36.

R. Bernardes, J. Cunha-Vaz, and P. Serranho (2012). Optical Coherence Tomography: a Concept Review. In Biological and Medical Physics. R. Bernardes and J. Cunha-Vaz, Eds. Berlin, Heidelberg: Springer Berlin Heidelberg.

B. Bouma and G. Tearney (2002), Handbook of optical coherence tomography. Marcel Dekker, New York.

S. Didas and J. Weickert (2007), Combining curvature motion and edge-preserving denoising scale space and variational methods in computer vision, Lecture Notes in Computer Science, 4485, 568-579.

L.C. Evans and J. Spruck (1991). Motion of level sets by mean curvature I. J. Differential Geometry, 33, 635681.

L. Junqueira and J. Carneiro (2005), Basic Histology: Text \& Atlas (Junqueira's Basic Histology). McGraw-Hill Medical.

T. Lu, P. Neittaanmaki, and X.-C. Tai (1992). A parallel splitting up method for partial differential equations and its application to Navier-Stokes equation. RAIRO Math. Model. and Numer. Anal., 26, 673-708.

G. Plonka and J. Ma (2008), Nonlinear regularized reactiondiffusion filters for denoising of images with textures, IEEE Trans. Image Process, 17(8), 1283-1294.

H.M. Salinas, and D.C. Fernández (2007) Comparison of PDE-based nonlinear diffusion approaches for image enhancement and denoising in optical coherence tomography, IEEE Trans. Med. Imaging, 26(6), 761771.

P. Serranho, M. Morgado and R. Bernardes (2012) Optical Coherence Tomography: a concept review. In Optical Coherence Tomography: A Clinical and Technical Update. R. Bernardes \& J. Cunha-Vaz Eds., SpringerVerlag, 139-156. 